\section{THE ATIOLOGY OF}

BACILLARY DYSENTERY IN ASYLUMS.

By W. S. DAWSON, M.A., M.B. OXon., M.R.C.P. LOND., D.P.M.,

AND

IV. MOODIE, M.D. ST. AND., D.P.M., ASSISTAYT MEDICAL OFFICERS, OLAYBURY MENTAL HOSPITAL.

THE question of asylum dysentery is of such interest to those concerned with the care of the insane that from time to time much useful investigation has been undertaken in the hope of establishing its retiology. The present state of our knowledge may be summed up in the words of Tidy, who says, in his "Synopsis of Medicine," " No specific organism is present, but bacteriological and serological investigations have indicated bacilli of the Flexner group." The study of a small series of cases which occurred in Claybury Mental Hospital during the earlier months of this year has yielded such definite results that it appears worthy of special note.

In 1900 Mott and Durham made a comprehensive study of dysentery in Claybury, and, although they were unable to isolate any specific organism, they showed clinically and post mortem that it resembled the bacillary type of the disease rather than the amœbic. Many observers have from time to time claimed to have discovered amobæ in the stools of patients, but their results require confirmation. ${ }^{1}$ In spite of all efforts to control it, dysentery has in the past been the cause of a large amount of the sickness in asylums, as well as a considerable percentage of the mortality. Thus, during the year 1919 , in 80 county and borough asylums 1722 cases of dysentery were notified, and of these 384 , or 20 per cent., died, forming 3 per cent. of the total number of deaths.

Chart 1.

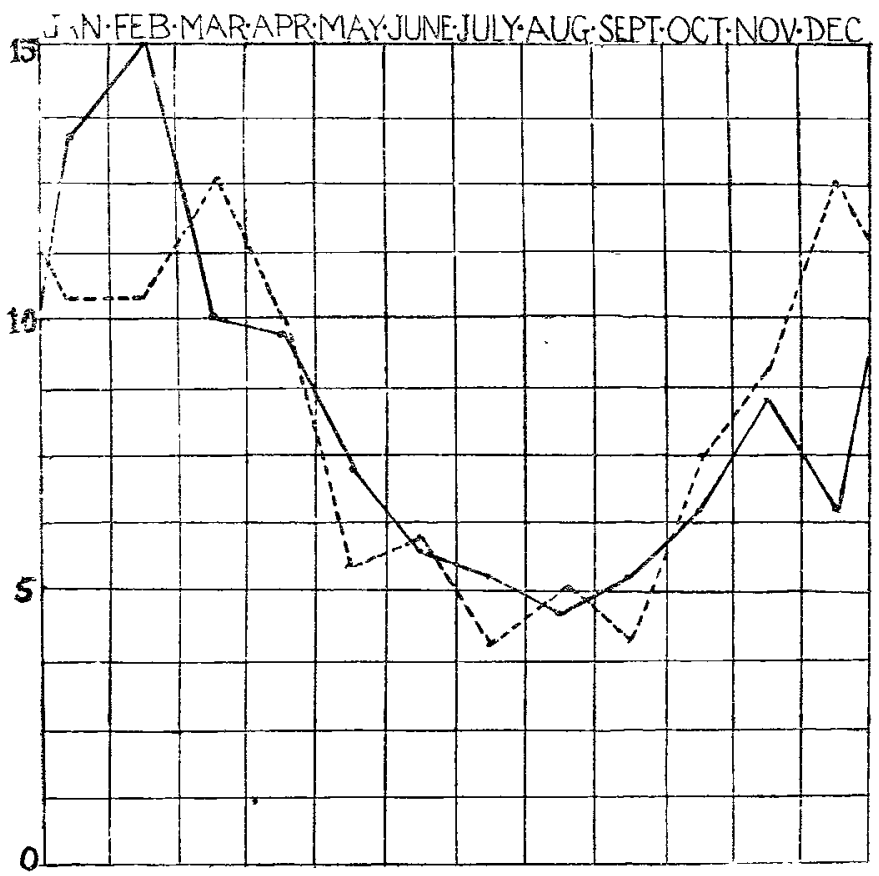

Continuous line shows monthly incidence of cases of dysentery in 80 county and borough asylums expressed as percentages of in 80 county and borough asylum line shows monthly incidence the total for 1919 . Interrupted line shows monthl incidence

Contrary to the accepted view that dysentery is a disease of hot weather and is generally spread by flies, the highest incidence of cases in Claybury occurs during the winter months. Chart 1 is constructed from a monthly average of cases occurring in the female wards during the years 1909 to 1920 . The published statistics of the Board of Control for all the asylums of England and Wales show roughly a similar incidence. ${ }^{2}$ Figures from the Ministry of Pensions and the Army for 1920, kindly supplied to

${ }^{2}$ Report of the Board of Control for 1919 , Part I. us by the Ministry of Health, show a remarkable similarity to our own, over $50 \mathrm{per}^{2}$ cent. of the total number of cases being reported during the period of January to April. It would therefore appear that asylum dysentery spreads not so much through flies as through some more direct method from patient to patient, especially during the winter months, when the patients are confined within doors for a greater number of hours per day on account of climatic conditions.

\section{Carriers.}

It is usually taken for granted that certain patients who have suffered from dysentery become carriers, and are therefore capable of disseminating the disease for a more or less protracted period after the acute stage has passed. One of our patients, who has relapsed seven times in eight years, is probably a carrier. She has been froe from acute symptoms for several months, and examination of her fæees at the present time fails to reveal the presence of an organism of dysentery. On the other hand, light transient attacks, which may pass unnoticed in a previously healthy paicient, may yet be of great danger as a source of infection. For exampie, a patient who was confined to bed for mental reassons on one occasion passed a loose motion in which a small quantity of blood was noticed. This was kept for examination, and produced a Flexner type bacillus on culture. The serum of this patient, at the end of the eighth week, agglutinated Prof. L. S. Dudgeon's antigen in a dilution of 1 in 125.

The question of relapsing cases who are probably carriers has been raised, and it is interesting to note in this connexion that of 590 patients who suffered from dysentery in Claybury during the past 12 years, 18 per cent. relapsed once, 6 per cent. twice, and 3 per cent. three times. In mild cases the dysentery bacillus disappears from the fæces in a few days, and the patient has apparently made a rapid recovery. We have, however, noted that not infrequently a relapse has occurred within a few weeks after the frees had been reported negative on several occasions. A patient who has had an attack of dysentery thus remains a possible source of infection to others, especially as mild, transient relapses may pass unnoticed in patients who are too demented to appreciate slight irregularities in their bodily functions.

To ensure the complete eradication of the disease from asylums, it would therefore appear necessary to carry out a very complete scheme of permanent isolation, and to treat every dysentery case as a potential permanent carrier. This principle is at present applied in Claybury, and all cases in the epidemic here reported occurred in wards where patients known to have previously suffered from dysentery were housed.

\section{Bacteriology.}

Seven cases occurred during the first four days of the outbreak, and from the fæces of all of these a bacillus of the Flexner type was cultivated. The patients affected were isolated in single rooms, and a strict watch was kept for any suspicious cases of diarrhoea, several of which occurred during the subsequent two months, and were investigated. During that time an identical bacillus was found in five more cases. The system of isolation of positive cases was persisted in, and, at the time of writing, no case of clinical or bacteriological dysentery has occurred for over a month.

The method employed in examining the fæces was as follows:- The specimen was taken from the motion and sent to the laboratory on a swab similar to a throat swab. This was found to be a very handy, clean, and efficient method of collecting specimens. If preferred, the swab may be introduced into the rectum of the patient, and the mucus from the wall of the rectum thus obtained directly. The latter method was employed extensively during the war, and was found to be excellent. It also has obvious practical advantages.

MacConkey's medium was used for plating, of a reaction of +4 acid. The reaction of the medium is $\mathrm{E} 2$ 
all-important, and must be adjusted with scrupulous care. Phenolphthalein was used as indicator in the titration, which was carried out at boiling point. The plates having been incubated for 24 hours, suspicious colonies were transferred to Russell's double sugar agar, in which Andrade's reduced fuchsin was used as indicator. On this medium it is usually possible to observe gas formation after 12 hours' incubation, and so to weed out the nondysentery organisms.

The growth obtained on Russell's medium was then asglutinated macroscopically on a slide with Dreyer's Oxford Flexner serum, the reaction, if positive, appearing in about 15 seconds. If agglutination appeared thus the case was treated as a positive. It was therefore possible to send out a laboratory report on the specimens of fæces well within 48 houss. This rapidity of diagnosis is extremely important from an epidemiological point of view. As a confirmatory test the organisms were subcultured and grown on the "sugars," and, in addition, were agglutinated macroscopically with Dreyer's Oxford serum to full titre. It was found in practice that no organism which passed the rough test failed to pass the more detailed and delicate one.

The bacillus was non-motile and Gram-negative, and, while producing acid without gas on glucose and mannite, it left lactose and saccharose unchanged. It is interesting to note that from two of the cases of diarrhoa from which the Flexner type bacillus was not isolated, B. pyocyaneus was obtained in almost pure culture. In the case of one patient who died, although there was extensive ulceration of the intestine reaching practically as high up as the duodenum, only cultivations from the rectal contents yielded the Flexner type bacillus, none being present in cultures taken from the higher parts of the intestine or the internal organs.

\section{Agglutination Tests.}

Frequent agglutination tests were carried out on the serum of all those patients whose fæces had been proved positive bacteriologically, and also on some cases whose freces did not yield positive results. Dreyer's standard Oxford antigen was used in all cases, and latterly another Flexner type antigen, kindly supplied to us by Prof. Dudgeon, of St. Thomas's Hospital, was used in addition.

Several facts of fundamental interest were collected. It was found that, with Oxford antigen, the first trace of agglutinins appeared at the end of the

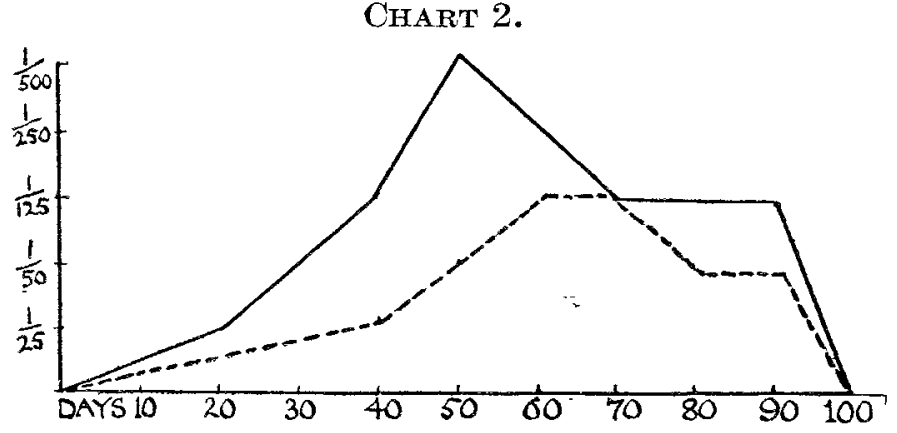

Agglutination titre at various perioda of the disease. Con tinuous line, with Dudgeon's antigen. Interrupted line, with Dreyer's antigen.

sixth week, and that agglutinins had disappeared at the end of the fourteenth week; while with Prof. Dudgeon's antigen agglutination began to appear about the beginning of the fourth week, and had disappeared at the end of the fourteenth week also. Maximum agglutination was obtained with Oxford antigen between the eight and tenth week, and with Prof. Dudgeon's antigen in the seventh week (Chart 2). No case in which the fæces were negative bacteriologically gave a positive agglutination with either antigen. Many agglutination tests were performed; using the organisms isolated from the cases themselves as antigen. The curves thus obtained conformed roughly with those given by Oxford antigen and that of Prof. Dudgeon.
Shaw Bolton and Nabarro ${ }^{2}$ have carried out extensive investigations on the agglutinins in dysentery. They state that a positive reaction occurs early in the disease, and that many apparently healthy persons, who are not known to have suffered from dysentery, give a positive reaction, thus raising the question of the possibility of unsuspected carriers. Our results are not in accordance with their findings.

\section{Conclusions.}

1. It is of paramount importance that the fæces of all cases of clinical dysentery and diarrhoea be examined in the laboratory. 2. Having regard to the fact that primary attacks and relapses may be so slight as to pass unnoticed, and the possibility of every case remaining a chronic carrier, very strict observation and a system of permanent isolation are necessary in order to prevent the spread of the disease. 3. Agglutinins are present in the blood of affected patients only after the fourth week of the disease, and therefore agglutination tests are of little diagnostic value.

\section{SEROLOGICAL TYPES OF PNEUMOCOCCI IN LOBAR PNEUMONIA.}

$$
\text { A STUDY OF } 100 \text { CASES. }
$$

\section{By FRED. GRIFFITH, M.B., Сн.B. VICT., D.P.H. (From the Pathological Laboratory of the Ministry of Health.)}

THE study of the immunological types of pneumococci has been shown by the workers of the Rockefeller Institute in America and by Lister in South Africa to be of great importance in the prevention and treatment of lobar pneumonia. The existence in this country of the types defined by the American workers had been established, but the information which has been published in respect to their distribution is still somewhat scanty. ${ }^{1}$ I therefore propose to contribute my observations on the serological characters of strains from 100 cases of lobar pneumonia which I have classified according to the American method. The standard type strains upon which the classification is based were presented by Prof. Flexner to Sir George Newman, who made them available for the purposes of this inquiry.

\section{Preparation of Monovalent Agglutinating Serums} in Rabbits.

Adult rabbits were used, being immunised by the intra venous inoculation of pneumococci in ascending doses. The suspensions of pneumococci were obtained by centrifuging broth cultures, the deposits from which were generally diluted with some uncentrifuged broth culture. The inoculations were made on three consecutive days and were followed by a rest of from five to seven days. For the first three series of inoculations the culture used was killed by heating to $60^{\circ} \mathrm{C}$. for half an hour, and was prepared freshly for each series. The immunisation was continued with living culture, beginning with a fraction of a cubic centimetre of broth culture and gradually rising until the whole of the deposit of $20 \mathrm{c.cm}$. of broth culture was administered at a dose. The total period of treatment was 10 weeks ; the animals were bled within 10 days after the last inoculation. The serums were tested upon pneumococci from broth culture suspended in salt solution, and gave for types 1 and 2 a titre of $1: 320$ and for type $31: 640$. These serums were re-tested after being preserved in a sterile condition in the ice-chest for 10 months; they showed no appreciable diminution in potency. No reaction with heterologous strains--e.g., type 1 serum against types 2 and $3, \& c$.- was obtained in dilutions of 1 in 20 .

In a second series of rabbits some observations were made on the progress of immunisation. Treatment commenced with suspensions of heated broth culture, preserved with 0.5 per cent. carbolic acid, and continued for five weeks inoculations being made on three successive days, followed by an interval of rest. The serums then showed titres ranging from $1: 20$ to $1: 40$ for the four type 1 rabbits, and $1: 4$ only for the three type 2 animals. The treatment was continued, but with a fresh heated suspension of each type;

A A report on this subject has been published by R. R. 